\title{
Competing Order and Asymmetric Tunneling Spectrum in High Temperature Cuprate Superconductors
}

\author{
Jiangping $\mathrm{Hu}$ and Kangjun Seo \\ Department of Physics, Purdue University, West Lafayette, Indiana 47907, USA
}

(Dated: November 29, 2018)

\begin{abstract}
We show that the asymmetric tunneling spectrum observed in the Cuprate superconductors stems from the existence of a competing order. The competition between the competing order and superconductivity can create a charge depletion region near the surface. The asymmetric response of the depletion region as the function of the external voltage causes the asymmetric tunneling spectrum. The effect is very general in a system which is near the phase boundary of two competing states favoring different carrier densities. The asymmetry which has recently been observed in the point-contact spectroscopy of the heavy fermion superconductor CeCoIn $\mathrm{I}_{5}$ is another example of this effect.

PACS numbers: 74.25.Jb, 74.25.Dw, 74.75.-h
\end{abstract}

\section{INTRODUCTION}

One important characteristic of the strongly correlated systems is the possibility of the existence of many competing orders. To identify these competing orders and study the interplay between them are two major tasks in this field. In high temperature cuprate superconductors, competing orders, such as magnetic $\stackrel{1.2}{=}$ stripe,,$\frac{3.4 .5}{,}$ d-density wave, $\underline{\underline{6}}$ microscopic current orders,$\underline{7.8}$ and so on, have been proposed. Although it is still a matter of debate, the competing orders have been widely considered to be responsible for the unusual properties and the universal phase diagrams of the materials. The competition between superconductivity and antiferromagnetism or other orders is also present in other strongly correlated electron systems such as heavy fermions.

In the cuprates, although the static global competing phases are rarely detected and the direct transition between the superconducting and the competing orders has not been observed, recent STM experiments have suggested that a static order may exist ${ }^{9.10 .11}$ The competing phases are expected to exist in the underdoped region. In the multiple layer cuprate materials, the middle layer is naturally underdoped ${ }^{12}$ In this case, the competition between the competing orders and superconductivity has been used to explain the behavior of Tc as a function of the number of layers $\frac{13}{}$ In this paper, we show that the carrier distribution close to the surface can also be naturally inhomogeneous along c-axis and the competing order is generically enhanced at the surface. The competition between the competing order and superconductivity can lead to an asymmetric tunneling spectrum. The effect could be the natural explanation of the asymmetry universally observed in the STM experiments in the cuprates 14 and the metal contact tunneling experiments $\frac{15}{5}$ in heavy fermion systems.

A particle-hole asymmetry is observed universally in different cuprate compounds. In traditional BCS type superconductors, tunneling spectrum is expected to be symmetric between negative and positive bias voltage. Such a large and universal asymmetry observed in high temperature superconductors remains a challenging puzzle to be solved. A comprehensive theory to understand the phenomena may be tied to the ultimate understanding of high temperature superconductivity since there are only a few universal features identified crossing different types of cuprate materials. A few theoretical ideas based on the $\mathrm{t}-\mathrm{J}$ model have been proposed $\stackrel{16.17 .18}{ }$

In this paper, we propose that the origin of this asymmetry is indeed tied to one fundamental physics in cuprates, the existence of the hidden competing order. We show that the asymmetry stems from the competing order at surface, which has been detected by the same STM measurement ${ }^{9.10 .11}$ When an external voltage is applied, the competing order near the surface can be weakened or strengthened depending on the sign of voltage. Therefore, the region of competing phases can be asymmetrically responded to the external field. This effect leads to asymmetric tunneling matrix elements. Such an effect is expected to be universal as soon as the competing orders favor different doping concentrations from the superconducting states. Interestingly, we notice that the similar effect indeed has been observed in tunneling experiments in heavy fermion systems ${ }^{15}$ The sample used in the experiment is also very close to the phase transition boundary between the AFM and SC orders.

In a semiconductor, charges can be accumulated on the surface due to the electronic surface states. In the bulk close to the surface, the space charges can be induced to screen the surface charge. This effect creates a space charge layer at the semiconductor interface. In the cuprates, even without electronic surface states, a natural charge depletion region near the surface can be created since the carriers are introduced stoichiometrically. The number of the carriers induced in the $\mathrm{Cu}-\mathrm{O}$ layer close to the surface from the neighboring layer A-O where A is $\mathrm{La}(\mathrm{Sr})$ in LSCO (BSCCO) can be very different from that inside the bulk. On the surface, free charges can be attached to the A-O plane to reduce the electrostatic energy and effectively reduces the doping concentration. Such an effect leads to the lower carrier density in the $\mathrm{Cu}-\mathrm{O}$ plane close to the surface. In Figure 1 we plot 
(a)

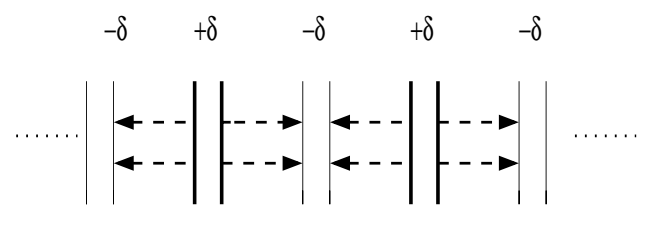

(b)

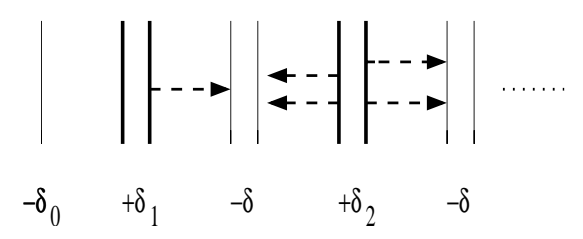

FIG. 1: Schematic picture of the carrier distribution and electrostatic field distribution along the c-axis in a double layer structure. The solid and darker solid lines represent the $\mathrm{A}-\mathrm{O}$ and $\mathrm{Cu}-\mathrm{O}$ planes respectively and the dash arrows represent the electrostatic field. (a) and (b) represent the distributions in the bulk and close to the surface respectively.

a schematic picture to show that in the presence of the surface, the electrostatic field can be reduced close to the surface to save the electrostatic energy which is the origin of the doping inhomogeneity in the layered compounds of the type $\mathrm{Tl}_{2} \mathrm{Ba}_{2} \mathrm{Ca}_{n-1} \mathrm{Cu}_{n} \mathrm{O}_{2 n+4}$ and related structures $\frac{12}{2}$ The effective carrier contribution from the A-O layer on the surface is diminished in order to save the electrostatic energy. As a result, the doping concentration on the $\mathrm{Cu}-\mathrm{O}$ layer close to the surface is lower than that in the bulk. Therefore, in the hole (electron) doped cuprates, a hole (electron) depletion region can be created near the surface. In the semiconductor, it is well known that an external voltage can change the space charge distribution near the surface and the depletion region has asymmetric response to an external electric field. Thus one can expect that similar effect happens in the cuprates, even though it is not clear how the asymmetric response could create a large asymmetric STM tunneling spectrum if the sample is simply in the superconducting state. In the presence of competing orders, however, the effect could be crucial. The variation of the carrier distribution could lead to a change of the configuration of the competing orders near the surface. In particular, when the competing orders are insulating, the tunneling amplitude can be significantly modified by the change of the configuration of the competing orders. Such an effect could be accounted for the observed asymmetric STM tunneling spectrum.

\section{MODEL AND FORMULATION}

We start to discuss about the physics in the structure shown in Figure 10 The total charge carrier energy near the surface is given by $F_{c}=\sum_{\langle i, j\rangle} V_{i j} \delta_{i} \delta_{j}$, where $\langle i, j\rangle$ is a pair of any layers and $V\left(x_{i}-x_{j}\right)$ are Coulomb energy between two layers if $i \neq j$ and band energy if

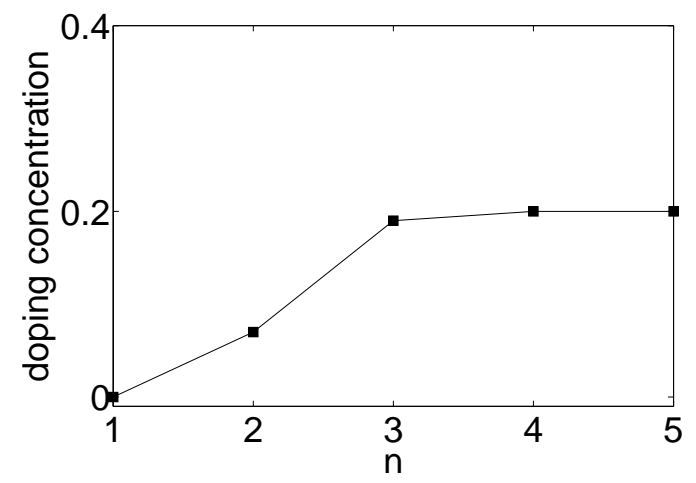

FIG. 2: The doping concentration near the surface. The average doping concentration inside the bulk is chosen to be 0.2 .

$i=j$. Since the physics is very general, we simply assume the sheet-charge model,${ }^{12}$ in which the uniform distribution of carriers in the $\mathrm{Cu}-\mathrm{O}$ plane forms two-dimensional sheets of charge. The charge in the A-O layers, except the one exposed on the surface, are fixed and determined by doping concentration. The charge in the layer at the surface can be freely adjusted since it is exposed to outside. Therefore, we can consider a uniformly doped superconductor with negative charge accumulated on the surface with density $-\delta_{0}$. This surface charge induces a non-uniform doping distribution of charges $\delta_{i}$ in the $i$ th $\mathrm{Cu}-\mathrm{O}$ plane. Then the band energy under the carrier distribution, $\rho_{i}=\delta_{i} / \delta$ along the $c$ axis can be written as

$$
U_{B}=\left(\pi \hbar^{2} / 2 m^{*}\right) \delta^{2} \sum_{i} \rho_{i}^{2},
$$

with $m^{*} \approx 4 m_{e} \frac{19}{19}$ And the electric field is $E(k)=$ $(4 \pi / \epsilon) \delta\left(1-\sum_{j}^{k} \rho_{j}\right)$, where $\epsilon$ is the background dielectric constant. The relevant electrostatic energy density is $U_{e}(i)=\left(e^{2} \epsilon / 8 \pi\right) \sum_{k}^{i} E_{k}^{2}$. The total charge carrier energy can be written as

$$
F_{c}=\frac{2 \pi e^{2} d_{0}}{\epsilon} \delta^{2}\left[\frac{1}{C} \sum_{i} \rho_{i}^{2}+\sum_{i}\left(1-\sum_{k}^{i} \rho_{k}\right)^{2}\right],
$$

where $C=4 m^{*} e^{2} d_{0} / \hbar^{2} \epsilon$ and $d_{0}$ is the distance between the adjacent planes. Using the values $d_{0}=11.69 \AA$ and $\epsilon \approx 12 \epsilon_{0}$, one obtains $C \approx 7.4$.

In the presence of a competing order, we adopt the Ginzburg-Landau free energy of a multi-layer system given in $\operatorname{Re}^{13}$,

$$
\begin{aligned}
F_{o}= & \sum_{j}\left[\alpha\left(\delta_{j}\right)\left|\psi_{j}\right|^{2}+\lambda\left|\psi_{j}\right|^{4}-\rho_{c}\left(\psi_{j} \psi_{j+1}^{*}+c . c .\right)\right. \\
& \left.+\alpha^{\prime}\left(\delta_{j}\right) \phi_{j}^{2}+\lambda^{\prime} \phi_{j}^{4}+g|\psi|_{j}^{2} \phi_{j}^{2}\right]
\end{aligned}
$$

where $\alpha\left(\delta_{j}\right)$ and $\alpha^{\prime}\left(\delta_{j}\right)$ are functions of the doping $\delta_{j}$ for each layer and all other parameters are assumed to be 
here is that
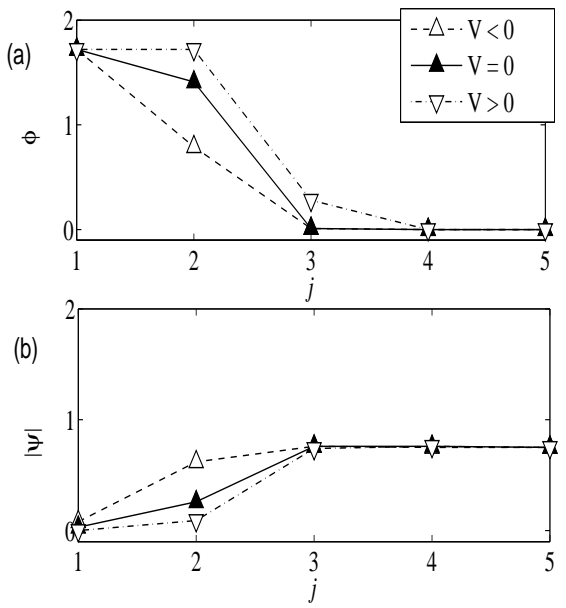

FIG. 3: The configurations of (a) the competing and (b) superconducting order parameters when the voltage is applied. $\mathrm{V}$ is the voltage dropped near the surface. The curves are calculated at $V=100,0,-100 \mathrm{meV}$ respectively. The absolute value of $\mathrm{V}$ is expected to be less than the bias voltage of the sample with respect to the tip.

constants. $\phi_{i}$ and $\psi_{i}$ are the competing order and superconducting order parameters on the $i$ th $\mathrm{Cu}-\mathrm{O}$ plane respectively. The total free energy of the system is given by $F_{t}=F_{c}+F_{o}$. For a multi-layer system, in general, we have to resort to numerical method to minimize the total free energy. However, as an approximation, one can minimize $F_{c}$ first with respect to $\delta_{i}$ and obtain the equilibrium charge distribution $\left\{\delta_{i}\right\}$. After obtaining the charge distribution by minimizing $F_{c}$, we can minimize $F_{o}$ with respect to $\psi_{j}$ and $\phi_{j}$ to find the configurations of the competing and superconducting order parameter as a function of layers. This simple approach is proved to be reliable since Coulomb energy is usually larger and only a few layer closest to surface are effected which we will show later. In fact, we have performed an independent numerical calculations to verify this by using of a variational Monte Carlo calculation to search the global minimum of the total free energy. The quantitative results for the distribution of charge density and order parameter configuration are only slightly different from the ones obtained through the above approximated approach.

Without external voltage, it is obvious that the A-O layer at the surface prefers zero charge density, i.e. $\delta_{0}=$ 0 , in order to minimize the charge energy. Therefore, the first $\mathrm{Cu}-\mathrm{O}$ near the surface will be underdoped in general. In the presence of external voltage, additional electric field can be built near the surface, which leads to an effective modification of the charge at the surface, i.e., $\delta(V)=\delta_{0}+f(V)$, where $f(V)$ is zero if $V=0$ and carries the same sign as the voltage $V$. Our natural assumption

$$
f(V)=c_{0} V .
$$

If we assume that the entire voltage is dropped in the competing order region which is roughly in one or two layers along c-axis, the value of $c_{0}$ can be estimated to be around $\frac{\epsilon a^{2}}{d_{0} e}$ which is $0.09\left(V^{-1}\right)$ for the lattice constant $a=4 \AA, d_{0}=11.69 \AA$, and $\epsilon=12 \epsilon_{0}$. In the underdoped superconductor $\left(\delta_{j}<0.2\right.$, for all $\left.j\right)$, we choose $\alpha\left(\delta_{j}\right)=$ $10\left(\delta_{j}-0.3\right), \alpha^{\prime}\left(\delta_{j}\right)=27\left(\delta_{j}-0.22\right), \lambda=\lambda^{\prime}=1$ and $g=1.2$. This choice of the parameters gives the right shape and the magnitude of the superconducting dome for the generic superconducting phase diagram ${ }^{13}$

\section{TUNNELING SPECTRUM}

The doping concentration at zero bias near the surface are calculated and shown in Figure 2 where the average doping concentration in the bulk is taken to be 0.2. This figure tells that only the first three layers closest to the surface are effected. The doping concentration recovers very quickly to the average values inside the bulk. The result also simplifies the numerical calculation to search a global minimum for the total free energy since only variables in a few layers need to be considered.

Figure 3 shows how the configurations of the order parameters respond to the applied voltage $V$, which is the voltage dropped near the surface. The curves in the figure are calculated at $V=100,0,-100 \mathrm{meV}$ respectively. The absolute value of $V$ is expected to be less than the bias voltage of the sample with respect to the tip. The positive $V$ corresponds to electrons tunneling into the sample. As the applied voltage increases from negative to positive, the competing order on the second and third layers increases whereas superconducting order decreases on the second layer. In the deep inside the sample, the superconducting order is uniform. Near the surface, however, the competing order parameter is dominant over the superconductivity. This means that the depletion region, where the competing order parameter $\phi$ is dominant, increases with the increasing voltage from negative to positive. The charge depletion region can be defined by the average depth of the competing order parameter, $l_{d}$,

$$
l_{d}=\sum_{j} j\left|\phi_{j}\right|^{2} / \sum_{j}\left|\phi_{j}\right|^{2} .
$$

In Figure 4 we plot the length of charge depletion region, $l_{d}$, as the function of the voltage. The doping concentration is chosen to be optimal.

In tunneling experiments, the tunneling matrix elements usually are assumed to be constant. However, the presence of the charge depletion region obviously leads to the variation of the matrix elements. The increase of the charge depletion region near the surface with the voltage bias $V$ implies the decrease of the conductance. Since the matrix elements are determined by the overlapping 


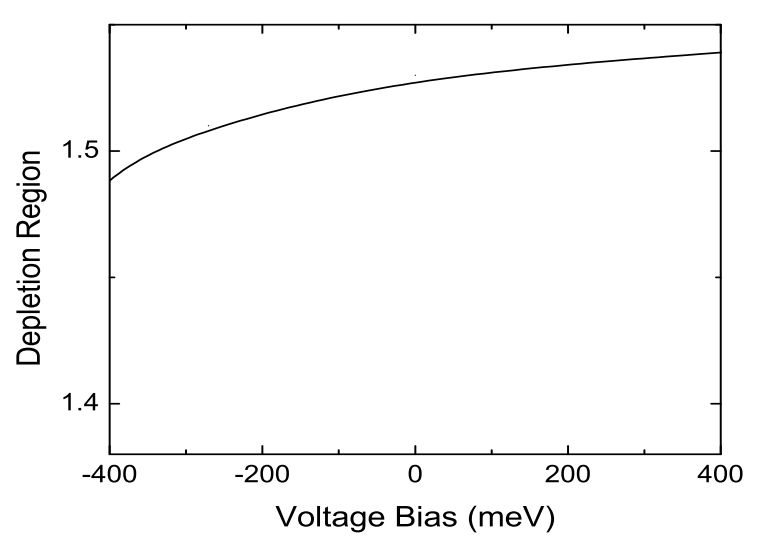

FIG. 4: The length of the charge depletion region as the function of the voltage bias. The doping concentration at bulk is set to be optimal.

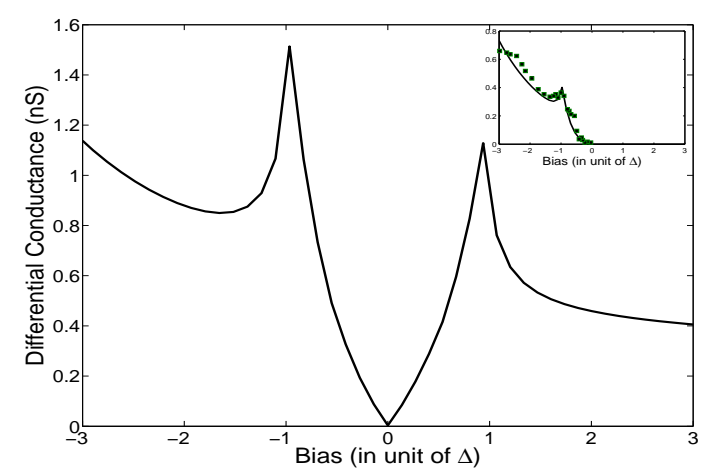

FIG. 5: The tunneling spectrum calculated by adding the effect of the depletion region in cuprates in superconductring states. The solid line in the inset shows the difference between the spectrum in negative voltage and one in positive voltage. The dashed dots are extracted experimental values from Ref ${ }^{14}$.

of wavefunctions between the tip and the measured material, the change of the competing order near surface can result in a significant variation of the tunneling matrix element. A slight variation of competing order near surface could lead to large change of the tunneling-matrix element.

The effect is universal as soon as a competing order exists close to superconducting phase, regardless of the nature of the competing order. The effect is large if the competing phase is insulating. One promising compet-

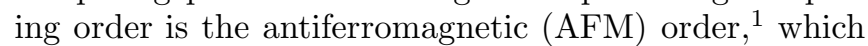
is an insulating phase. It has been suggested that the transition between AFM and SC orders are controlled by chemical potential,,$\stackrel{1,20}{?}$ which can be changed by the doping concentration. Moreover, it has also been argued that the superconducting materials are locked at the AFM and SC transition point in the large range of

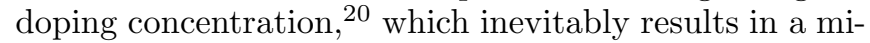

croscopic phase separation. In this case, near the surface, the material should be very sensitive to external electric field since it effectively changes the chemical potential. If the competing order is metallic, such as d-density wave (DDW),$\frac{6}{t}$ the effect can be small. The value of asymmetry is also expected to depend on the disorder which is generically present in the cuprates. Although it is hard to make an quantitative estimation since the different competing orders will lead to different magnitude of the asymmetry, we can make a reasonable assumption to obtain quantitative result to see whether the experimental result can be naturally explained.

In a simple approximation, we can assume that the depletion region plays as a tunnelling barrier in the STM measurement between the STM tip and the superconducting phase. The energy of the barrier is determined by the single-particle energy gap $E_{g}$. Since the depletion region is close to half filling, $E_{g}$ should be expected in order of $U$ in the microscopic Hubbard model. This approximation leads to an exponential dependence of the tunneling matrix element as a function of the length of the depletion region,

$$
G(V) \approx G_{0}(V) e^{-\frac{2}{\hbar} \sqrt{2 m^{*} E_{g}} l_{d}(V) d_{0}},
$$

where $G_{0}(V)$ is the tunneling conductance for a d-BCS state which is assumed to be symmetric for negative and positive bias voltages i.e. $G_{0}(V)=G_{0}(-V), d_{0}$ is the lattice constant along c-axis and $l_{d}(V)$ is the depletion length in the unit of lattice constant in c axis. In Figure 5 we calculated a typical spectrum following the above approximation. The asymmetry between positive and negative bias voltage, $G(-V)-G(V)$ is also shown in the inset. Amazingly, the experimental data can be fitted to this crude approximation. The dotted points in the inset of figure 5 are the extracted experimental values from Ref ${ }^{14}$ Using the result of $l_{d}(V)$ calculated by Equation (5), there is only one fitting parameter $E_{g}$. For the experimental result 14 indeed in the order of $U$. Therefore, this result shows that the suppressed superconducting order parameter by the dominance of competing order parameter close to the surface of the sample can explain the asymmetry of the tunneling conductance background of high temperature superconductors.

\section{CONCLUSION AND DISCUSSION}

This theory provides several explicit predictions. First, there is an onset temperature for the tunneling asymmetry since there is onset temperature for the competing order. In cuprates, a natural onset temperature could be $T^{*}$ associated with the pseudogap. We expect that the tunneling asymmetry disappears above $T^{*}$. Secondly, the tunneling asymmetry should be larger if the bias voltage is larger. It is a monotonically increasing function 
of the voltage, which is consistent with the experimental result of the tunneling asymmetry as the function of voltage. The experimental result shows that the asymmetry is small in low voltage and becomes larger and larger as the voltage becomes large 14 The theory predicts that there could be a large enhancement of the asymmetry at high bias voltage if the nature of the transition between the competing order and superconductivity is the first order transition. For example, the transition between AFM and SC is typically first order. Finally the asymmetry which has recently been observed in the point-contact spectroscopy of the heavy fermion superconductor $\mathrm{CeCoIn}_{5}{ }^{15}$ can also be naturally explained by this effect since the material in this experiment is close to the boundary of the AFM and SC phase transition. Our theory predicts that the asymmetry should be weakened when the material moves away from the boundary toward superconducting zone by tuning the pressure.

In conclusion, we have presented that in the presence of competing orders at surface, the tunneling matrix elements can strongly depend on externally applied voltage. This effect can lead to a natural explanation of the tunneling asymmetry observed in the STM experiments. We have shown that this effect could exist universally in materials where the superconducting phase is close to a competing order and our prediction can be easily tested experimentally.

The authors would like to thank S. Chakravarty, S. Kivelson, S. C. Zhang, E. W. Carlson, Congjun Wu and H.D. Chen for valuable discussions. J. P. Hu would like to thank W. K. Park for extremely useful discussion about his experiments. This work is supported by Purdue research funding.
1 S. C. Zhang, Science 275, 1089 (1997).

2 S. Sachdev, Rev. Mod. Phys. 75, 913 (2003).

3 S. A. Kivelson, E. Fradkin, and V. J. Emery, Nature (London) 393, 550 (1998).

4 J. Zaanen, Nature (London) 404, 714 (2000).

5 E. W. Carlson et al., Concepts in High Temperature Superconductivity (Spinger-verlag, 2004), review chapter to appear in 'The Physics of Conventional and Unconventional Superconductors' ed. by K. H. Bennemann and J. B. Ketterson.

${ }^{6}$ S. Chakaravarty, R. B. Laughlin, D. K. Morr, and C. Nayak, Phy. Rev. B 63, 094503 (2001).

7 P. A. Lee and X.-G. Wen, Phy. Rev. B 63, 224517 (2001).

8 C. M. Varma, Phy. Rev. Lett. 83, 3538 (1999).

9 J. E. Hoffman, E. W. Hudson, K. M. Lang, V. Madhavan, H. Eisaki, S. Uchida, and J. C. Davis, Science 295, 466 (2002).

10 T. Hanaguri, C. Lupien, Y. Kohsaka, D. H. Lee, M. Azuma, M. Takano, H. Takagi, and J. C. Davis, Nature
(London) 430, 1001 (2004).

11 M. Vershinin, S. Misra, S. Ono, Y. Abe, Y. Ando, and A. Yazdani (2004).

12 M. DiStasio, K. A. Muller, and L. Pietronero, Phy. Rev. Lett. 64, 2827 (1990).

13 S. Chakravarty, H. Y. Kee, and K. Volker, Nature (London) 428, 53 (2004).

14 S. H. Pan, E. W. Hudson, K. M. Lang, H. Eisaki, S. Uchida, and J. C. Davis, Nature (London) 403, 746 (2000).

15 W. K. Park, L. H. Greene, J. L. Sarrao, and J. D. Tompson, cond-mat/0409090.

${ }^{16}$ W. Rantner and X.-G. Wen, Phy. Rev. Lett. 85, 3692 (2000).

17 P. W. Anderson and N. P. Ong, cond-mat/0405518.

18 J. E. Hirsch, cond-mat/0407642.

19 L. Krusin-Elbaum, R. L. Greene, F. Holtzberg, A. P. Malozemoff, and Y. Yeshurun, Phy. Rev. Lett. 62, 217 (1989).

20 S. C. Zhang and J. P. Hu, Phy. Rev. B 60, 13070 (1999). 